\title{
Consequences of fragmentation for the ability to adapt to novel environments in experimental Drosophila metapopulations
}

\author{
J. Bakker • M. E. C. van Rijswijk • F. J. Weissing • \\ R. Bijlsma
}

Received: 8 September 2009/Accepted: 12 January 2010/Published online: 6 February 2010

(C) The Author(s) 2010. This article is published with open access at Springerlink.com

\begin{abstract}
We used experimental populations of Drosophila melanogaster, which had either been subdivided (metapopulations) or kept undivided for 40 generations, to study the consequences of population subdivision for the tolerance and adaptive response after six generations of exposure to novel environmental factors (high temperature, medium with ethanol or salt added) for traits with different genetic architectures. In this setup, we attempted to separate the effects of the loss of fitness due to inbreeding (i.e., the survival upon first exposure to stress) from the loss of adaptive potential due to the lack of genetic variation. To place our experimental results in a more general perspective, we used individual-based simulations combining different options of levels of gene flow, intensity of selection and genetic architecture to derive quantitative hypotheses of the effects of these factors on the adaptive response to stress. We observed that population subdivision resulted in substantial inter-deme variation in tolerance due to redistribution of genetic variation from within demes to among demes. In line with the simulation results, the adaptive response was generally lower in the subdivided than in the undivided populations, particularly so for high temperature. We
\end{abstract}

J. Bakker and M. E. C. van Rijswijk contributed equally to this work.

J. Bakker $(\bowtie) \cdot$ M. E. C. van Rijswijk · R. Bijlsma

Population and Conservation Genetics, University of Groningen, PO Box 14, 9750 AA Haren, The Netherlands

e-mail: joke.bakker@rug.nl

J. Bakker - M. E. C. van Rijswijk - F. J. Weissing - R. Bijlsma

Theoretical Biology, University of Groningen, PO Box 14,

9750 AA Haren, The Netherlands

J. Bakker - M. E. C. van Rijswijk

Evolutionary Genetics, University of Groningen, PO Box 14,

9750 AA Haren, The Netherlands observed pronounced differences between stress factors that are likely related to the different genetic architectures involved in resistance to these factors. From a conservation genetics viewpoint, our results have two important implications: (i) Long-term fragmentation in combination with restricted gene flow will limit the adaptive potential of individual subpopulations because adaptive variation will become distributed among populations rather than within populations. (ii) The genetic architecture of the trait(s) under selection is of great significance to understand the possible responses to novel stresses that may be expected.

Keywords Adaptive potential .

Drosophila melanogaster - Experimental metapopulation . Stress tolerance $\cdot$ Population subdivision

\section{Introduction}

To meet environmental challenges, it is crucial that a population is able to cope with the imposed stress at short term, and to evolve adaptive adjustments on a longer time scale. To ensure the short-term survival of the population, albeit at a reduced fitness level, some minimum number of individuals has to tolerate the stressful conditions sufficiently to enable reproduction and to sustain the population at lower numbers. The ability to tolerate stress is largely determined by two factors: phenotypic plasticity and/or the presence of (partially) resistant genotypes (Bijlsma and Loeschcke 2005). Even if the population survives the stress, it will have a reduced fitness. To ensure long-term survival the population needs to adapt to the new environment. Rapid adaptation to novel environments requires the availability of ample genetic variation (Macnair 1991; Lynch and Lande 1993). Since both stress tolerance and 
adaptation mostly occur on short to intermediate evolutionary time scales, new beneficial mutations will be rare, hence, the initial adaptive response will mainly depend on the standing genetic variation (Bijlsma and Loeschcke 2005; Orr and Unckless 2008; Dionne et al. 2009). This has recently been clearly demonstrated by Bell and Gonzalez (2009) who showed that a yeast population could only escape extinction on a lethal concentration of salt, when the population was sufficiently large to contain resistant individuals. Thus, the availability of genetic variation is crucial for the persistence of populations exposed to changing environmental conditions, and mostly determines the adaptive potential of a population.

The availability of genetic variation may be compromised, however, if environmental deterioration is associated with habitat fragmentation. In fact, subdivision of a formerly large population into small population fragments or demes in combination with limited gene flow may have profound consequences for the availability of genetic variation. Genetic drift will become more prominent in small populations, resulting in the loss of genetic variation from single demes and increased differentiation among demes within the entire metapopulation (Wright 1951). In other words, genetic variation is redistributed from the within-deme level to the among-deme level, hereby substantially reducing the adaptive potential of single demes (Whitlock 2002; Swindell and Bouzat 2006). Furthermore, small populations are prone to inbreeding and accompanying inbreeding depression that often causes populations to be more sensitive to environmental deterioration (Crnokrak and Roff 1999; Bijlsma et al. 2000; Reed et al. 2002, 2003, 2007; Armbruster and Reed 2005; Kristensen et al. 2008), which decreases fitness and increases the risk of population extinction (Saccheri et al. 1998; Bijlsma et al. 2000; Frankham 2005).

Thus, whereas exposure to deteriorating environmental conditions requires an adequate response, the prerequisites for such a response appear to be severely hampered by the results of habitat fragmentation. As a consequence, research into the adaptive response of subdivided populations to environmental challenges is of major importance for conservation biology. Recent studies on plants, butterflies and Drosophila have shown that the adaptive response generally decreases with increasing levels of inbreeding, e.g., due to a pronounced reduction of the effective population size (Frankham et al. 1999; Whitlock and Fowler 1999; Saccheri et al. 2001; Reed et al. 2003; Swindell and Bouzat 2005, 2006; Briggs and Goldman 2006). However, except for a few (Frankham et al. 1999; Willi and Hoffmann 2009), most of these studies involve the adaptive response for quantitative characters that under natural conditions are not, or only peripherally, related to fitness.

This study aims to investigate the adaptive response to environmental deterioration of subdivided populations using Drosophila melanogaster metapopulations as a model system. We used two types of populations that differed in their history of fragmentation. Six populations had been subdivided for 40 generations with gene flow through migration at a relatively low level ("metapopulations"), and these were matched by more or less similar-sized, undivided control populations. This provided us with a unique opportunity to investigate the effect of population subdivision on the adaptive response to changing environments. We subjected both the subdivided and the undivided populations for six generations to different environmental stress factors, and measured the survival at first exposure to the stress factors (i.e., stress tolerance), as well as the increase in survival after six generations of exposure to the stress factors (i.e., adaptive potential).

Adaptation to stress environments can presumably be regulated by different genetic architectures for different stress factors. Therefore, we used the three stress factors: high temperature, salt and ethanol. In large outbred populations, the tolerance to high temperature has most often been observed to be a polygenic character (Cavicchi et al. 1995; Loeschcke et al. 1997), but a genotype-by-environment interaction may occur in the form of conditional deleterious alleles that are only expressed at high temperatures (Vermeulen and Bijlsma 2004; Kristensen et al. 2008; Vermeulen et al. 2008). Tolerance to saline environments is regulated by many genes with small effects (i.e., entirely polygenic, Wallace 1982). In case of tolerance to ethanol, the alcohol dehydrogenase gene Adh is involved as a major gene, although several other genes also play a role (Chakir et al. 1996; Malherbe et al. 2005).

We focused on the following questions: (i) What is the effect of habitat fragmentation (i.e., small populations connected by limited gene flow) on stress tolerance of a population? (ii) What is the effect of fragmentation on the adaptive potential of a population? (iii) Is the response to stress different if the underlying genetic architectures differ?

In addition, we ran individual-based computer simulations to place our experimental results in a more general perspective. We combined different options of genetic architecture with a range of increasing selection strengths and a range of increasing levels of gene flow to derive quantitative hypotheses of the effects of genetic architecture and population fragmentation on stress tolerance and adaptive potential.

\section{Materials and methods}

Population history

The populations of $D$. melanogaster used in this study were part of long-term experiments investigating the effects of 
genetic drift, gene flow and local extinction in small subdivided populations that used genetic markers to visually monitor allele frequencies over time (unpublished data). All populations were founded with flies from two marker stocks obtained from the Umeå stock centre with either red-brown eyes $\left(b w^{75} ; s t\right)$ or white eyes $(b w ; s t)$ in homozygous individuals. Prior to all experiments, the two marker stocks had been crossed and maintained as a mixed population for several generations to homogenize the genetic background. As such, we expect linkage disequilibrium to be minimal except for the genomic region around the marker loci. We then used all heterozygous orange-eyed $\left(b w^{75} / b w ; s t\right)$ flies to initiate six subdivided metapopulations with six demes each holding 50 individuals per deme, and six large undivided populations with 300 individuals per population.

Each deme of a metapopulation was maintained in a glass vial containing $18 \mathrm{ml}$ of standard medium [ $26 \mathrm{~g}$ dead yeast, $54 \mathrm{~g}$ sugar, $17 \mathrm{~g}$ agar and $13 \mathrm{ml}$ nipagin solution (10 g nipagin in $100 \mathrm{ml} 96 \%$ alcohol) per litre], whereas the undivided populations were maintained in $125 \mathrm{ml}$ bottles containing $30 \mathrm{ml}$ of the same medium. All populations were kept in the same climate room at $25^{\circ} \mathrm{C}$, $40-60 \% \mathrm{RH}$ and $24 \mathrm{~h}$ of light.

The six metapopulations (M1-M6) each comprised of $n=6$ demes (Fig. 1) with an approximate size of $N=50$ individuals (harmonic mean over 40 generations: 57.9, $56.2,56.1,51.0,52.5,50.7)$ each (unpublished data). In these metapopulations, adult flies were allowed to migrate between the demes for 12-24 h, approximately 3 days after eclosion. In three of the metapopulations (M1-M3) flies migrated according to a circular stepping-stone pattern (i.e., migrants move between adjacent demes only, Fig. 1, right). In the other three metapopulations (M4-M6) flies migrated according to an equidistant $n$-island ("migrant-pool") pattern (i.e., migrants first assemble in a central pool, and then move on into any of six demes, Fig. 1, left). After migration, the individuals from each deme were transferred to fresh vials to lay eggs for the next generation. The average number of immigrants was 0.5 and 1.3 for migrant-pool and stepping-stone migration, respectively. The number of migrants was estimated in a parallel series of metapopulations that included extinctions and is based on the observed colonization rate of empty subpopulations in this series (data not shown).

The undivided populations (P1-P6) were each maintained in a single bottle with an approximate population size of $N=220$ individuals (harmonic mean over 40 generations: $233.8,208.1,225.3,216.0,199.1,228.0$ ). Although we initially aimed to reach on average the same total population size as in a metapopulation, ca 300 , this was unfortunately not realised. Every 2 weeks the newly
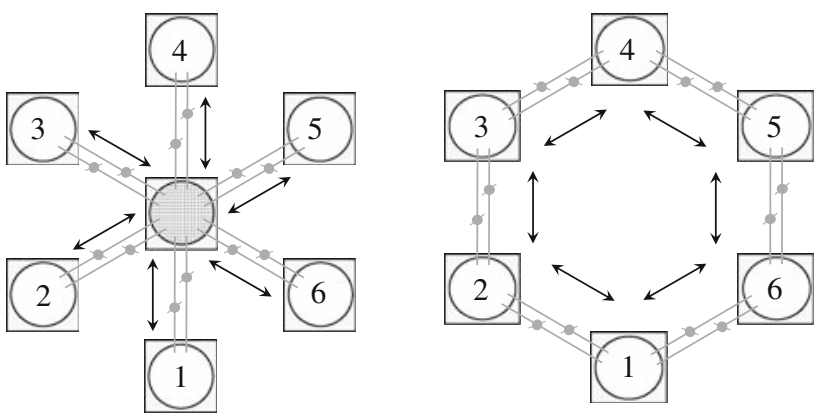

Fig. 1 Setup of experimental D. melanogaster metapopulations in two spatial configurations. A metapopulation consists of six demes represented by compartments connected through tubes that can be opened or closed for migration by taps. Each deme is either connected to all other demes via a central compartment (left) representing a migrant pool structure, or connected directly to two adjacent demes (right) in a circular stepping-stone pattern. Each compartment holds a vial containing medium and a fly population, whereas the central compartment in the migrant pool configuration holds a vial containing only medium that is closed on top by a piece of nylon wire mesh. Migrating flies can move in both directions as indicated by the arrows

emerged adults were transferred into a fresh bottle to lay eggs, and discarded after 1-2 days of egg-laying to maintain discrete, uncrowded generations.

All populations were continued for 40 generations, after which we set up the flies from all demes and all undivided populations as $(36+6)$ separate lines maintained in two bottles each (ca 250 individuals per bottle, individuals from the two bottles were mixed each generation). We increased the population size to minimize the effects of genetic drift on the genetic variation present after 40 generations of subdivision. We maintained the lines at standard conditions for five generations until the start of the adaptation experiment. Unfortunately, we lost two lines from metapopulation M4 due to a bacterial infection, reducing the total number of lines to $(34+6)$.

\section{Adaptation experiment}

To initiate the adaptation experiment, flies from all lines were allowed to lay eggs in two bottles in the different stress environments: high temperature $\left(28-28.5^{\circ} \mathrm{C}\right)$, salt medium (3\%) and ethanol medium (10\%). In addition, we maintained all lines in two bottles at standard conditions as controls. Individuals from the two bottles were again mixed in each new generation. Egg-laying occurred for approximately 2 days, after which we discarded the parents to maintain discrete generations. In each generation, we initially collected the adults emerging in the stress environments in bottles at standard conditions, and transferred them to the stress environments for the next generation after most of the adults had emerged. 


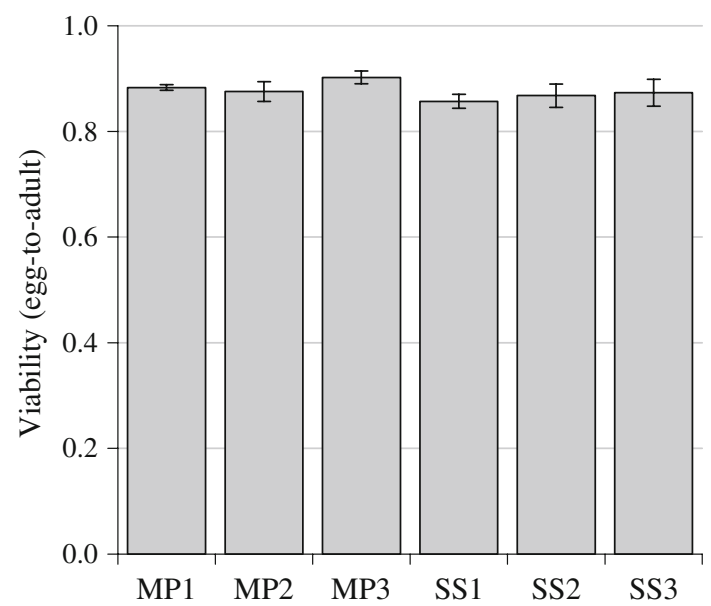

Fig. 2 Viability in the benign control environment. The bars show the average viability $( \pm \mathrm{SE})$ for each metapopulation. MP1-MP3 are the migrant-pool metapopulations (Fig. 1, left) and correspond to metapopulations M4-M6 in the remainder of this paper; SS1-SS3 are the stepping-stone metapopulations (Fig. 1, right) and correspond to metapopulations M1-M3

We kept the stress treatments at a moderate level aiming at an egg-to-adult survival of at least $25 \%$ to prevent the extinction of lines. However, given that the replicates differed considerably in tolerance and that it was logistically impossible to treat replicates separately, the actual survival rates generally varied between 20 and $60 \%$ egg-to-adult survival. This varied also between generations. On average, the stress intensity was higher for temperature stress (where often backups had to be used, see below) than for salt stress where egg-to-adult survival rates varied between 40 and $60 \%$.

For the high temperature treatment, we assessed the viability at $28.5 \pm 0.5^{\circ} \mathrm{C}$, but we maintained a backup at $28^{\circ} \mathrm{C}$ during the adaptation process. Due to small fluctuations in the climate room the temperature sometimes became $29^{\circ} \mathrm{C}$, which can induce male sterility (David et al. 1983; Rohmer et al. 2004). In these cases, the backup at $28^{\circ} \mathrm{C}$, which never suffered from sterility, was used to start the next generation. This backup procedure was necessary at least once for each line due to the random fluctuations, and up to four times for lines that performed very poorly at high temperatures already in the first generation (see results).

After six generations of stress treatment, we maintained the lines at standard conditions for two more generations before assessing viability in order to obtain sufficient individuals, to synchronize the generations and to avoid carry-over effects. This procedure may, however, have resulted in some relaxation of selection.

To determine the level of adaptation of each line, we tested both the original, unadapted lines and the adapted lines in the stress environments. We allowed ca 40 females of each line to lay eggs for 5-10 h on standard medium, and we then carefully transferred the eggs to vials containing stress medium, or to vials that were placed at high temperature. We set up six replicates of 50 eggs each for all lines. After emergence, we counted all flies to calculate the egg-to-adult viability per line.

Normally, one would estimate the viability cost of exposure to stress relative to the viability under benign conditions. However, as the experiment was quite laborious already, we did not do this. In addition, a previous test showed that the viability under benign conditions was very high, i.e., around the maximum possible under the applied experimental conditions, and no significant differences were observed between (sub)populations (Fig. 2). Moreover, when we used these data to correct the experimental data the results were found to be qualitatively the same (data not shown). As these control data were obtained more than ten generations before the actual experiment we decided not to correct the viabilities observed under stress relative to the data observed under benign conditions.

Since our data were not normally distributed, either before or after standard transformations, we used KruskalWallis tests to analyze differences in viability between the metapopulations (average over the six demes) and the undivided populations, with Dunn-Şidak corrections to adjust the significance levels for multiple testing (Sokal and Rohlf 1995). For the subdivided populations we used the average over six demes as we sampled subdivided and undivided populations at different levels. To test for the effects of stress, subdivision and their interaction at the population level (i.e., six metapopulations versus six undivided populations), we applied the Scheirer-Ray-Hare extension of the Kruskal-Wallis test that enables a nonparametric two-way ANOVA with replication (Sokal and Rohlf 1995). All tests were performed with Statistix (version 8.0).

\section{Individual-based simulations}

Simulations allow us to systematically evaluate the effects of subdivision on the adaptive response for a wide range of relevant parameters. The resulting framework creates baseline expectations that facilitate the interpretation of the experimental results. In addition, we used simulations to generate expectations on the variance in fitness before and after adaptation to stress conditions for different levels of gene flow, varying from minimal (i.e., completely isolated demes) to maximal (i.e., an undivided population with the size of a metapopulation) levels and different selection pressures. 
The simulation model mimics the experimental setup (i.e., 40 generations of subdivision in a standard environment followed by six generations of exposure to a stress environment) and is parameterized for the mating system of Drosophila melanogaster (i.e., lottery polygyny, female remating, variation in female reproductive success). The impact of stress is implemented as viability selection in the zygote life stage. We assume an additive model with selection coefficient $s$ and per-locus viabilities $1,1-s / 2$ and $1-s$ for the genotypes $A A, A a$ and $a a$ in case of nonlethal alleles. For a recessive lethal allele, the viabilities are 1,1 and 0 , respectively.

In the initial stage of a simulation run, we let both the metapopulations and the undivided populations evolve for 40 generations in the absence of selection and with gene flow through migration in the metapopulations as in the original experimental setup. Since previous simulations never yielded large differences between migrant-pool and stepping-stone migration (unpublished data), we only looked at migrant-pool migration. We used the experimental average deme size $N=50$, but we used the size of an entire metapopulation, $N=6 \times 50=300$ for the undivided populations instead of the experimental average size of undivided populations that turned out lower $(N=220)$ than planned, since the primary aim of the simulations is to provide insight into the relevant parameter space rather than mimicking the experiment exactly. Furthermore, preliminary simulation runs indicated that the differences between results for population sizes 220 and 300 are an order of magnitude smaller than the variation between replicate runs for a given population size. In the final stage, we increased the size of each deme to $N=300$ and disabled migration (cf. setting up the experimental lines), and we subjected all resulting "lines" to viability selection for six generations as in the adaptation experiment.

We implemented four different genetic architectures comprising either polygenic traits regulated by few (5) or many (20) loci, or traits regulated by one major gene with "normal" additive variation or a recessive lethal allele at a single locus. For the single gene traits, the selection coefficients of $s=0.1, s=0.4$ and $s=0.7$ represent additive selection of increasing strength, while recessive lethals correspond to $s=1$. For the polygenic traits, the $k$ loci are unlinked with equal selection coefficients $s_{k}=s / k$ per locus. Fitness is calculated multiplicatively across loci (Hedrick 1994), and is similar to the corresponding values of the single gene traits. We ran simulations for a range of migration rates $m(0,0.1 / N, 1 / N, 10 / N$ and 1$)$, regarding the undivided populations as subdivided populations with unlimited migration, i.e., $m=1$. We ran 1,000 replicate runs of a single metapopulation adding up to 6,000 replicate demes, or 6,000 replicate runs of undivided populations.

\section{Results}

In the Fig. 3 we summarize the average viabilities in all lines before adaptation (Fig. 3, left column) and after adaptation (Fig. 3, middle column). The migrant-pool metapopulations did not differ significantly from the stepping-stone metapopulations with regard to viability (Kruskal-Wallis tests, adjusted $\alpha=0.0085$ ) for either of the three stress factors, both before (high temperature: $H=0.98, P=0.32$, salt: $H=0.17, P=0.68$, ethanol: $H=0.45, P=0.50$ ) and after (high temperature: $H=0.17, P=0.68$, salt: $H=5.2, P=0.023$, ethanol: $H=0.064, P=0.80)$ adaptation. The single significant difference for salt stress after adaptation becomes nonsignificant upon correction for multiple testing. Visual inspection of the data (see also Fig. 2) confirms that the spatial configuration of the metapopulations (island versus stepping-stone) had no systematic effect on the outcome of the experiments. In the rest of this paper we therefore pool the results of both spatial configurations into the single category "metapopulation" (i.e., subdivided populations as opposed to undivided populations).

\section{Viability before adaptation}

As all lines showed a similar fitness in the control environment, we assessed the viability at first exposure as a measure of the tolerance to a novel environment that is the prerequisite for an adaptive response to the environmental challenge. The viability in the stress environments varied greatly around values of ca 0.5 and 0.4 for the subdivided and undivided populations, respectively (Fig. 3, left column, first three panels), a substantial reduction when compared to the average value of 0.87 obtained under standard benign conditions (Fig. 2). Particularly for high temperature stress, the variation among demes within a metapopulation was large (see large SE's) with averages ranging from near zero to $0.6-0.8$ in some cases. Hence, the initial variation in tolerance among demes was already extremely large for this particular stress. These differences are mostly significant even after correction for multiple testing (Fig. 3). Hence, population subdivision has resulted in increased variation in tolerance to the novel environments among demes. Increased genetic drift in the small demes resulting in genetic differentiation among the demes within a metapopulation might explain this large variation. The large variation among demes (ranging from near $100 \%$ mortality to more than $80 \%$ survival) for the high 
High temperature

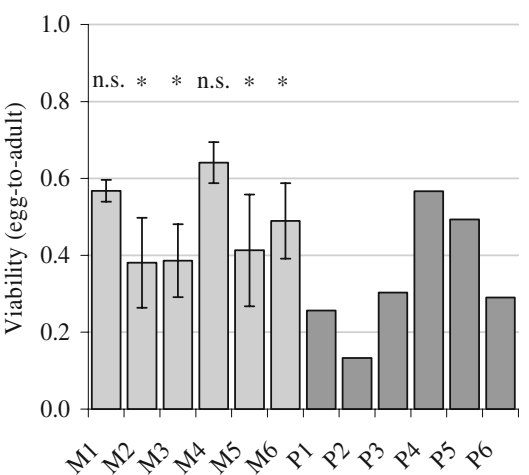

Salt

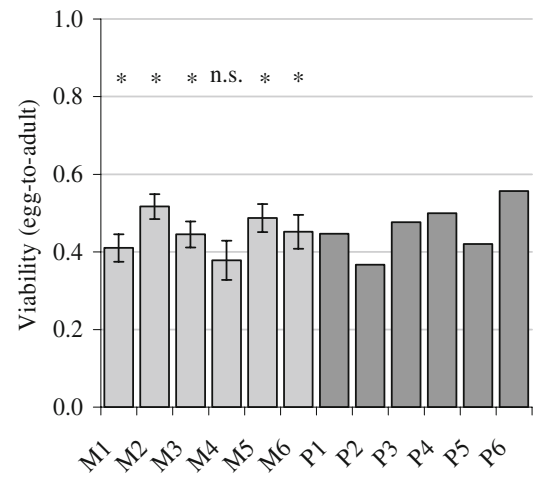

Ethanol
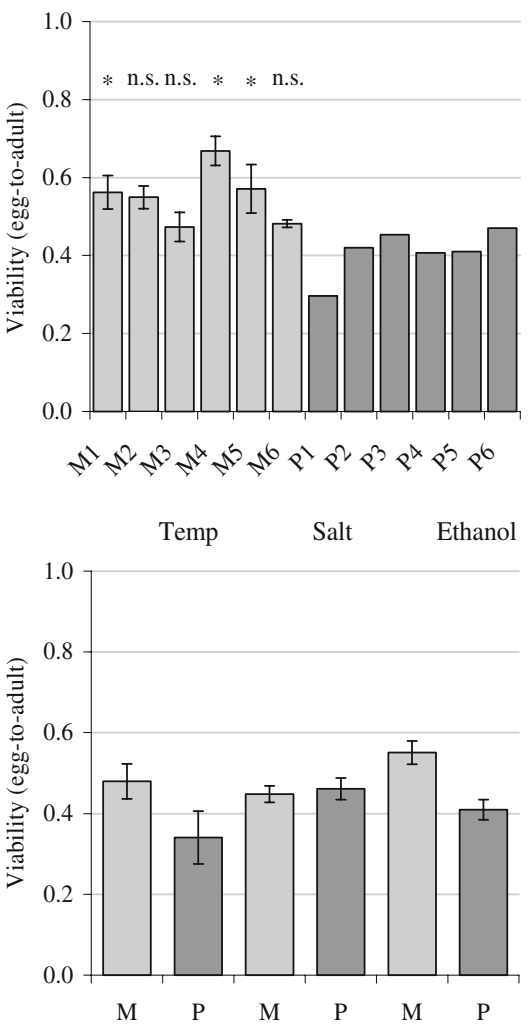

High temperature

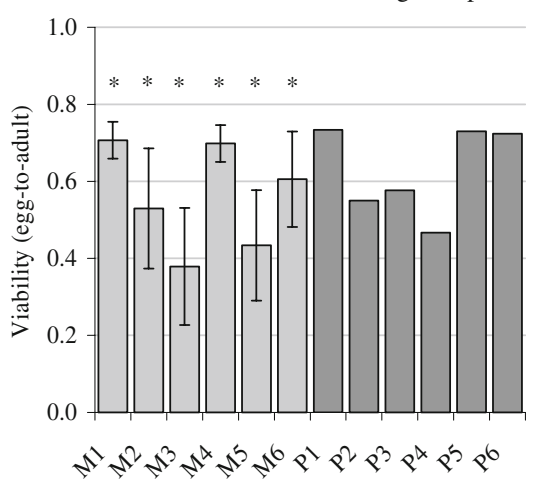

Salt

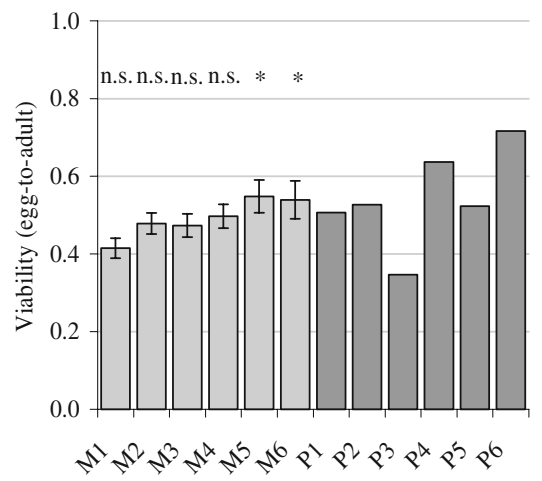

Ethanol
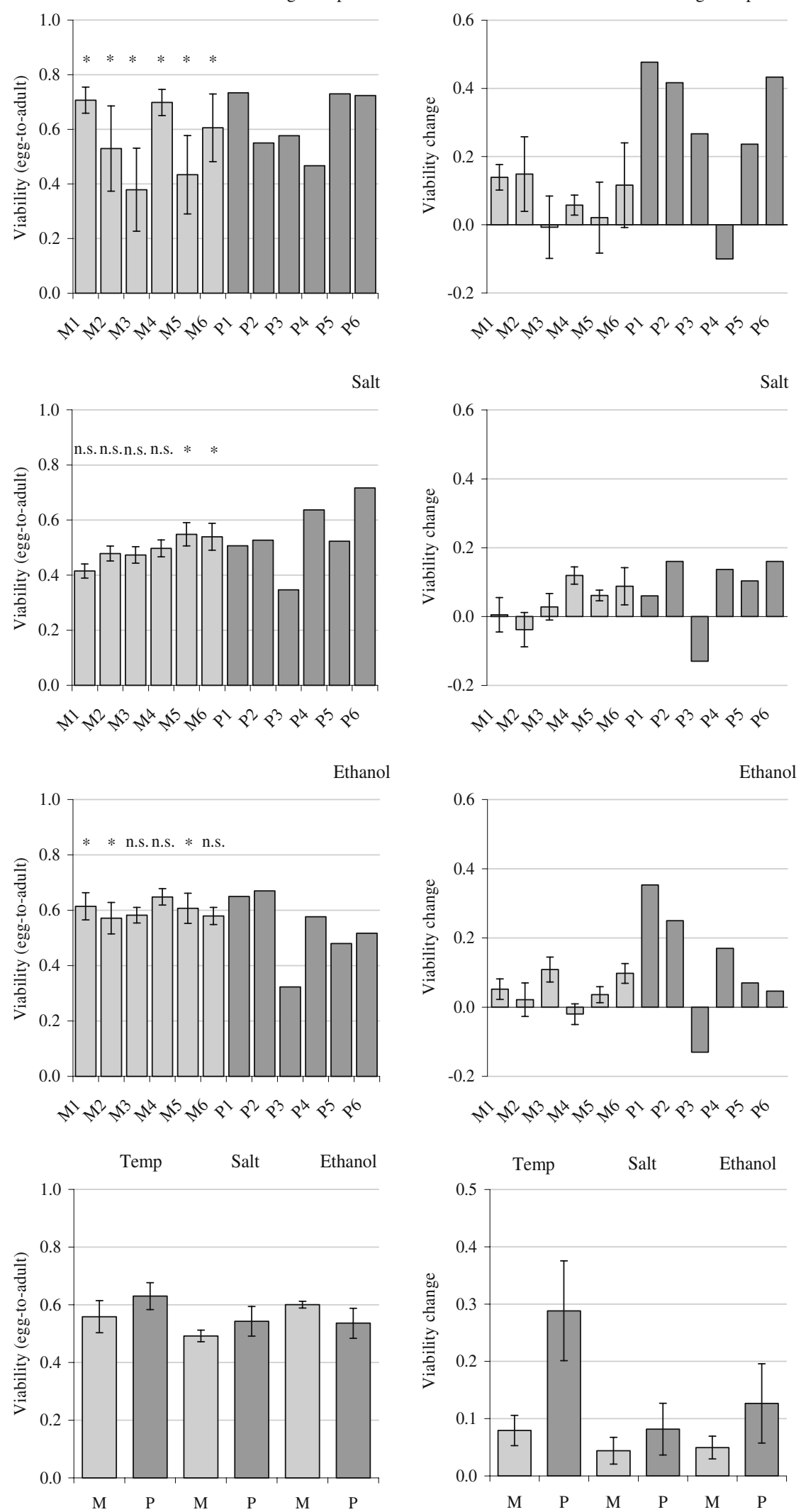

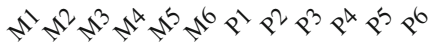

thanol 
4Fig. 3 Viability in three stress environments (i) before adaptation (left column), (ii) after six generations of adaptation (middle column), and (iii) the change in viability as a result of the six generations of adaptation (right column). The three upper rows show the average viability in the three stress environments high temperature, salt stress and ethanol stress, respectively. The bars show the average for each of the six subdivided metapopulations (M1-M6, light grey) and for each of the six undivided populations (P1-P6, dark grey); the error bars depicted for the metapopulations are SE's based on the averages for the six demes. The bottom row shows the viability (or viability change, right panel) averaged over the six metapopulations (light grey bars) and the six undivided populations (dark grey bars) for each of the three stress environments. * indicates a significant difference in viability among the demes within a metapopulation (upper rows, Kruskal-Wallis tests, adjusted significance level $\alpha=0.0028$ ), while n.s. means a non-significant difference

temperature stress may reflect the presence of conditionally detrimental alleles approaching fixation in some demes while the same alleles may have been lost from other demes due to genetic drift, as they are neutral under benign conditions.

As the possible presence of highly detrimental alleles being only expressed at $29^{\circ} \mathrm{C}$ was totally unexpected, we recently tested the original marker stocks that were at the basis of all experimental populations for the detrimental effect. The viability of the two marker stocks $b w^{75}$;st and $b w$;st was determined at both 25 and $29^{\circ} \mathrm{C}$ (five replicates of 100 eggs for each stock and each treatment). The results (mean viability $\pm \mathrm{SE}$ ) for $b w^{75}$;st were $0.67 \pm 0.15$ and $0.51 \pm 0.03$ for 25 and $29^{\circ} \mathrm{C}$, respectively. For $b w$;st viability was $0.71 \pm 0.03$ at $25^{\circ} \mathrm{C}$, but this dropped to $0.09 \pm 0.07$ at $29^{\circ} \mathrm{C}$. This clearly shows that the $b w ; s t$ stock carries one or more detrimental alleles that are only expressed at $29^{\circ} \mathrm{C}$. Initial crosses (unpublished data) have shown that the lethal effect is recessive.

We use the average of the six demes within a metapopulation to analyze the tolerance at the level of entire metapopulations versus that of undivided populations (Fig. 3, left column, bottom panel). The difference is only significant for ethanol (Kruskal-Wallis tests, adjusted $\alpha=0.017$, high temperature: $H=2.1, P=0.15$, salt: $H=0.10, \quad P=0.75$, ethanol: $H=8.3, \quad P=0.004$ ). Interestingly, the tolerance to ethanol is higher in the subdivided than in the undivided populations. Comparable with the among-deme level of variation, the variation among populations is by far the highest for high temperature stress.

\section{Viability after adaptation}

After six generations of exposure to the stress environments, we assessed the viability again to detect potential changes due to adaptation. On average, the viability has increased (Fig. 3, middle column), but the variation among demes is still considerable. The variation among demes in tolerance to high temperature has not decreased but seems to have become even more pronounced, with viabilities that either have increased to near maximum, or are found to be near zero (compare SE's of Fig. 3, left and middle panels). This suggests that most probably a detrimental allele has become fixed in the demes where the viability is low. These demes barely survived six generations of exposure to high temperature, and their viability did not only not increase, but occasionally even decreased, which may be simply due to experimental variation. In such cases, we often had to rely on the backups kept at $28^{\circ} \mathrm{C}$ to prevent extinction. High levels of male sterility at temperatures above $28.5^{\circ} \mathrm{C}$ in inbred lines might have presented an additional problem in the high temperature treatment (Rohmer et al. 2004).

At the metapopulation level, the differences between subdivided and undivided populations have become smaller than those observed before adaptation (Fig. 3, middle column, bottom panel), and they are not significant for any of the stress environments (Kruskal-Wallis tests, adjusted $\alpha=0.017$, high temperature: $H=1.6, P=0.20$, salt: $H=0.92, P=0.34$, ethanol: $H=0.42, P=0.64$ ).

\section{Change in viability}

The right column in Fig. 3 shows the extent of adaptation to the stress environments during the six generations of exposure. We observe substantial differences among the demes within the metapopulations with respect to the change in viability, again particularly for the high temperature stress. In some demes the viability readily improved, whereas in others no improvement occurred at all. Notwithstanding the large variances, we see that for each of the stresses ten out of 12 populations (divided and undivided populations combined) show on average an increase in viability after exposure to the stress for six generations (Sign test $P<0.02$ ). This indicates that our adaptation scheme was successful, even though we observe a high level of variation both within and among populations, which makes statistical inferences difficult.

At the metapopulation level (Fig. 3, right column, bottom panel), the increase in viability was always more pronounced in the undivided than in the subdivided populations. However, given the large variation observed, these differences are not significant when testing each stress environment separately (Kruskal-Wallis tests, adjusted $\alpha=0.017$, high temperature: $H=3.7, P=0.055$, salt: $H=1.6$, $P=0.20$, ethanol: $H=1.3, P=0.26$ ). Testing the three environments combined in a Kruskal-Wallis ANOVA indicates that a significant part of the variation between metapopulations and undivided populations is due to subdivision $(H=5.87, \quad \mathrm{df}=1, \quad P=0.02), \quad$ whereas 
$\mathrm{s}=0.1$

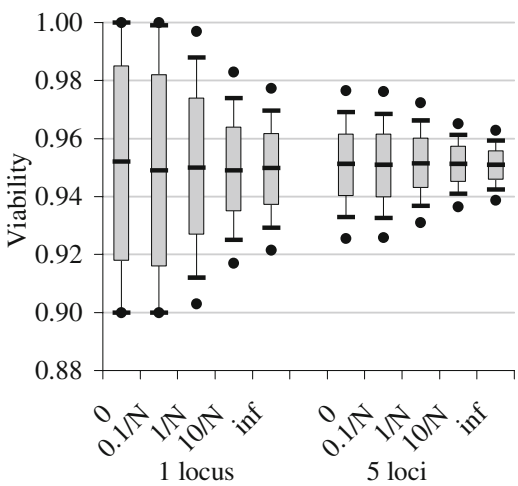

$\mathrm{s}=0.4$

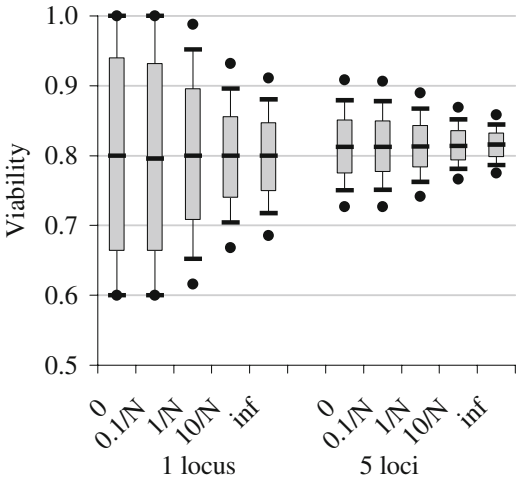

$\mathrm{s}=0.7$

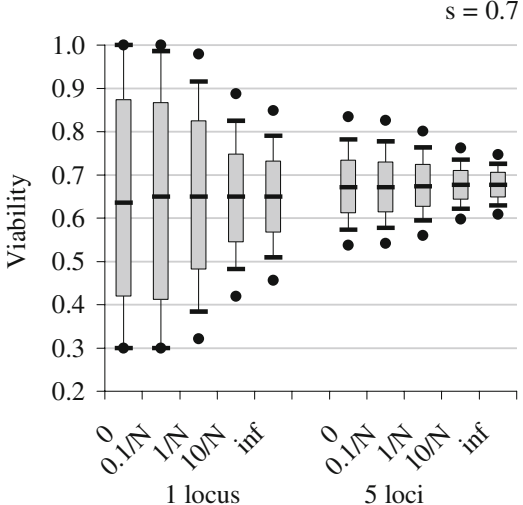

recessive lethal

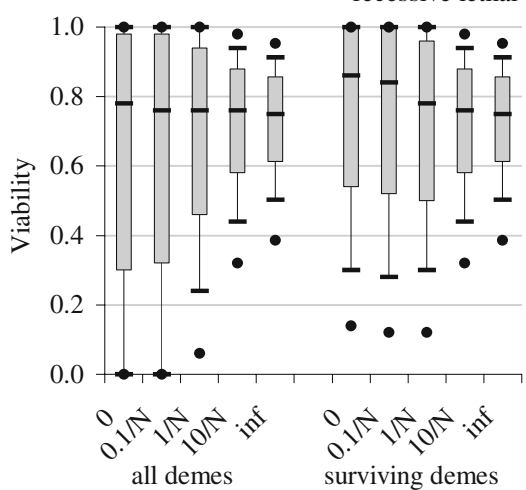

$\mathrm{s}=0.1$
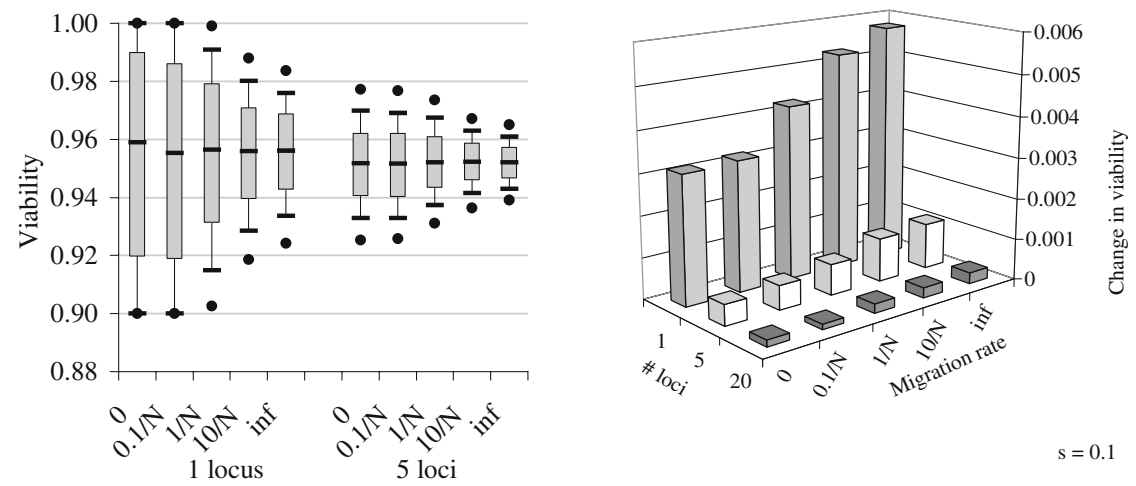

$\mathrm{s}=0.1$
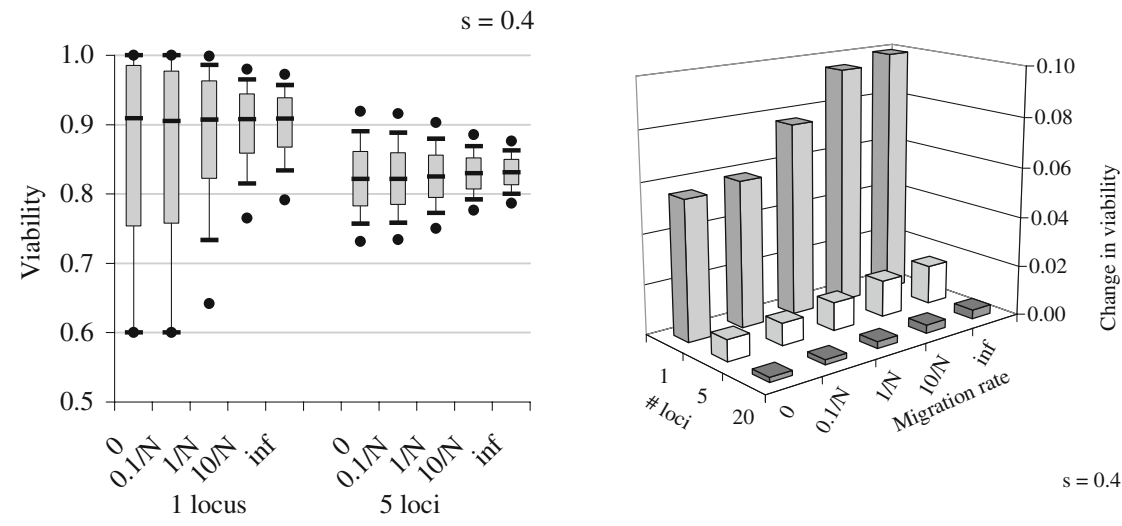

$\mathrm{s}=0.4$
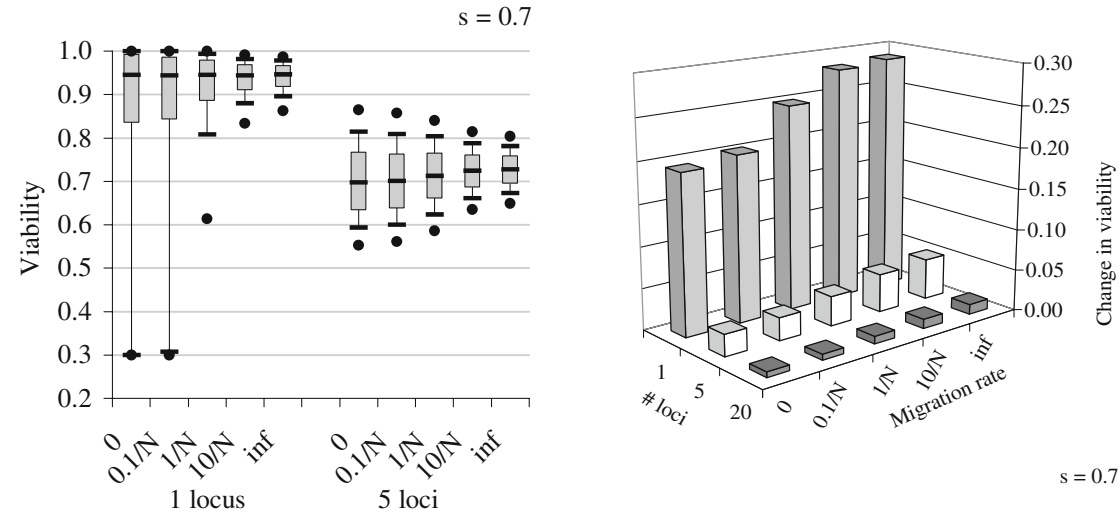

$\mathrm{s}=0.7$

recessive lethal

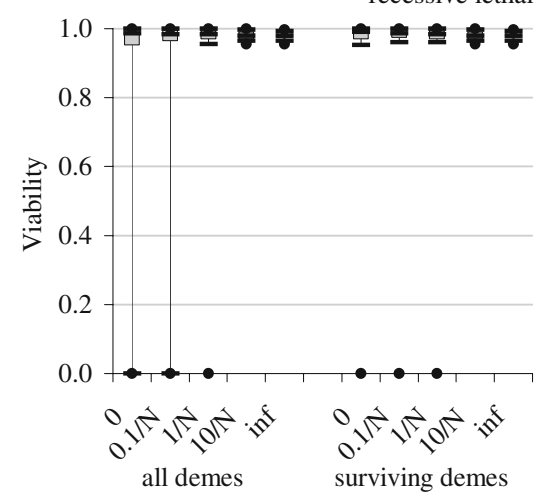

recessive lethal

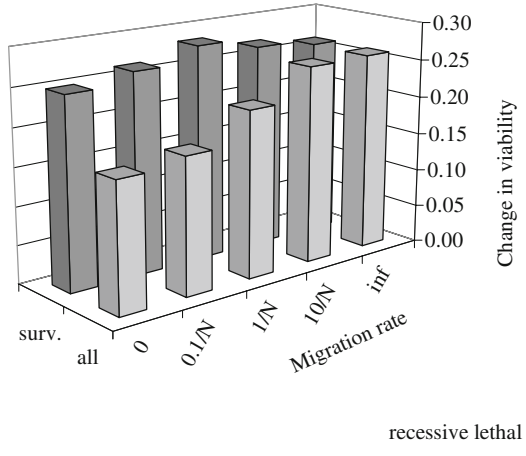


4Fig. 4 Computer simulations indicating the expected effect of population subdivision on initial tolerance to stress (left column) and to tolerance after six generations of adaptation to a stress environment (middle column). The first three rows display the results for increasing (top to bottom) selection strength $(s=0.1,0.4,0.7)$. Each panel shows the results of decreasing levels of subdivision $(m=0,0.1 / N, 1 /$ $N, 10 / N$ and 1) for two genetic architectures (major gene and polygenic trait). Bars: interquartile range with median, whiskers: $75 \%$ range, dots: $90 \%$ range. The right column shows the expected effects of subdivision on the adaptive response (i.e., the change in viability) after six generations of exposure to a stress environment for increasing selection strength for all three genetic architectures [major gene (light grey), polygenic traits regulated by five (white) and 20 (dark grey) loci, respectively]. The bottom row shows comparable results for strong selection against a recessive lethal allele. The results for the adaptive response are analysed for all demes (light grey), and excluding demes with zero initial tolerance (dark grey, see text for details)

environment $(H=2.97$, df $=2, P=0.23)$ and the interaction $(H=0.31$, df $=2, P=0.86)$ were not significant.

Individual-based simulations

Figure 4 shows the expected effect of population subdivision on stress tolerance (left column) and adaptation to a stress environment (middle column), and the impact of degree of subdivision ( $m=0,0.1 / N, 1 / N, 10 / N$ and 1$)$, strength of selection $(s=0.1,0.4,0.7)$ and genetic architecture (one major gene, polygenic traits regulated by five and 20 loci) (right column). The results for 20 loci (not shown) are very similar to the results for five loci, but the quantitative differences in viability are even smaller, as selection will take longer to arrive at a similar outcome when increasing numbers of loci are involved. The bottom row of Fig. 4 shows the expected effect of subdivision on tolerance (left) and adaptation (middle) for the special case of selection against a recessive lethal. We can distinguish three main trends. First, the average viability does not change when migration rates increase, but the variation in viability among demes decreases substantially (Fig. 4, left column). This shows that high migration rates are more efficient in mitigating the effect of local genetic drift on the differentiation among demes within a metapopulation. Second, the efficiency of the adaptation process increases considerably with increasing strength of selection, as indicated by the increase of the median values (Fig. 4, middle versus left column). Third, the variation becomes lower as the number of loci regulating a trait increases, since the extreme phenotypes are rare when large numbers of unlinked loci with small effects per locus affect a trait (Macnair 1991). In the special case of the recessive lethal (Fig. 4, bottom row, left and middle plots), a considerable number of demes obviously became extinct at the start of the adaptation process in populations with little or no migration. In these demes, the conditionally lethal allele has become fixed; hence, the tolerance to the stress factor is zero. Excluding the extinct demes from the analyses (right plots) suggests that almost all populations will rapidly attain maximum fitness due to very strong selection resulting in near complete removal of the recessive deleterious allele.

The right column of Fig. 4 summarizes the expected effects of six generations of adaptation on viability with regard to population subdivision, strength of selection and genetic architecture. The resulting increase in viability is qualitatively similar for all three selection strengths, but the magnitude of the change increases with increasing selection intensity. For polygenic traits the selection intensity per locus is low, hence the more loci are affecting the trait, the longer it will take to substantially increase viability, and thus, to properly adapt to a new environment. For a single locus, limited gene flow within a population may result in allele fixation, and thus, in the lack of adaptive potential, in a considerable number of demes, explaining the relatively large effect of the degree of subdivision on the increase of viability. The results for the single-locus eye-colour marker used in the experimental populations (unpublished data) confirm that allele fixation within demes commonly happens when migration rates are low. The bottom right panel of Fig. 4 shows similar results for the recessive lethal as for the single locus trait, in particular when all demes are considered (light grey bars).

In many cases, viability is not an additive trait. Therefore, we have also run simulations with fitness values of 1 , 1 and $1-s$ for genotypes $A A, A a$ and $a a$, respectively. However, the results and responses turned out to be qualitatively the same (data not shown). At the lower selection coefficients $(0.1$ and 0.4$)$ the response even tended to be somewhat lower than for the additive selection model. This may seem contradictory; however, one has to realize that the initial tolerance (before adaptation) is somewhat higher under the dominant selection model than under the additive selection model.

\section{Discussion}

Our study experimentally investigates the effects of population subdivision on the adaptive response for traits with various genetic architectures. Most studies testing the effects of genetic bottlenecks and a subsequent loss of genetic variation on the potential to adapt to new environments have used traits that are not or only indirectly related to fitness. Studies on plants, butterflies and Drosophila using morphological traits showed that the adaptive potential decreases with increasing bottleneck severity (Whitlock and Fowler 1999; Saccheri et al. 2001; Kristensen et al. 2005; Swindell and Bouzat 2005; Briggs and Goldman 2006, but 
see also Bryant et al. 1986; Bryant and Meffert 1996; Ruano et al. 1996; Cheverud et al. 1999). Fitness-related traits often suffer from inbreeding depression, which may seriously complicate the results as it may be difficult to separate extrinsic (environmental stress) and intrinsic (genetic stress) causes. Frankham and co-workers (Frankham et al. 1999; England et al. 2003) used time to extinction to study the adaptive potential of bottlenecked populations of Drosophila exposed to increasing salt concentrations. They found a negative correlation between the severity of the bottleneck and the time to extinction under increasing stress levels. However, they did not attempt to separate the effects of the loss of fitness due to inbreeding (i.e., the survival upon first exposure to stress) from the loss of adaptive potential due to the lack of genetic variation. Nevertheless, it is clear from all these studies that the loss of genetic variation due to genetic bottlenecks can be a significant limiting factor for adaptation under changing conditions. Hence, we would expect lower levels of tolerance and adaptive potential for the metapopulations than for the undivided populations in our experimental setup, since population fragmentation might easily have resulted in genetic bottlenecks for single demes. We found additional evidence for such bottlenecks based on the results of the single-locus eyecolour marker, and on a corresponding analysis of Adh allozymes (unpublished data).

Based on our simulation results, we also expect the variation in viability to be lower among the undivided populations (unlimited migration, $m=1$ ) than among the subdivided populations (low migration, $0.5 / N<m<1.3 / \mathrm{N}$ ), and to increase from salt stress (polygenic) to high temperature stress (mostly polygenic) to ethanol stress (major gene) for our experimental setup. After six generations of adaptation, we would expect a high response to ethanol stress, but lower response to high temperature and salt stress because of their polygenic character. However, the observed response to ethanol stress turned out to be more in line with the predictions for a polygenic architecture, whereas the high but variable response to high temperature stress points at the presence of one or more recessive lethals acting as major genes.

\section{Initial tolerance}

The tolerance to ethanol was on average lower for the undivided populations than for the metapopulations. This is contrary to the simulation results (Fig. 4, left column) indicating that different levels of gene flow affect only the variation in viability, but not the average viability at first exposure. Although the experimental conditions differed between the metapopulations and the undivided populations (demes were kept in vials and undivided populations were kept in bottles), the uniformly high viability under standard conditions (Fig. 2) indicates that both types of population were optimally adapted to the standard laboratory environment prior to the current experiment. In practice, other factors than the genetic make-up, which were not included in the simulations, may affect the variation at a locus, e.g., some (unintentional) variation in fly densities, or development time affecting variation at the Adh-locus (Van Delden and Kamping 1979).

First exposure to the stress environments resulted in substantial variation in tolerance among the demes. In line with the expectations based on the simulations, the variation is smallest for tolerance to salt, which is a polygenic trait. The variation in tolerance to ethanol is less than might be expected for a trait regulated by a single major gene. Although the Adh gene is involved with tolerance to ethanol as a major gene, other genes play a role as well (Chakir et al. 1996; Malherbe et al. 2005), hence ethanol resistance may behave more like a polygenic trait regulated by a small number of loci. The simulations predict lower levels of variation for such a system. Resistance to high temperature is generally regarded to be a polygenic trait (Cavicchi et al. 1995; Krebs et al. 1996; Loeschcke et al. 1997) and based on micro-array expression patterns many genes and processes are changing under short heat shocks (Sorensen et al. 2005). However, recessive highly detrimental alleles that are expressed at higher temperatures are also well documented in Drosophila (Lindsley and Grell 1968; Suzuki 1970) and extracted from natural populations (Dobzhansky et al. 1955; Tobari 1966). Such recessive, highly detrimental alleles that are only expressed at high temperatures represent an extreme case of this genotype-by-environment interaction, and are relatively frequent (Oudman et al. 1991; Bijlsma et al. 1999; Vermeulen and Bijlsma 2004). The high variation in tolerance to high temperature most probably results from the presence of the conditionally expressed detrimental allele that was observed in the bw;st marker stock. As such, we argue that the tolerance to high temperature stress is here governed by one or a few gene(s) with large fitness effects, thus resembling a trait regulated by a major gene, and its dynamics would more agree with a recessive lethal.

The results for stress tolerance imply in general that substantial amounts of genetic diversity may be preserved in subdivided populations, although the distribution among demes, and hence the tolerance level per deme, will vary considerably. In other words, the available genetic variation previously present within demes became redistributed to variation among demes within a metapopulation due to population subdivision, resulting in greatly increased variation in initial tolerance among demes. 


\section{Adaptive response}

The change in viability after six generations of adaptation to environmental stress conditions is not significantly different between the metapopulations and the undivided populations for the individual stresses, although the simulations predict a higher adaptive response for higher levels of gene flow, i.e., when populations are more connected. For the salt and ethanol environments, the response in either type of population is small (5-10\%) considering the relatively large selection intensity that initially allowed only $50 \%$ of the individuals to survive. In line with these observations, the simulation results (Fig. 4, right column) also indicate that the adaptive response should generally be very small for polygenic traits. The low response in the ethanol treatment may be partly explained by the fact that $10 \%$ ethanol may exert only little selection when the flies lay eggs directly on the food (Bijlsma-Meeles 1979). As such, the system for ethanol might have reacted as a polygenic system rather than a single locus system. The response to high temperature is quite substantial for the undivided populations, in line with the presence of a conditional lethal, but is on average not significant because of the large variation among the six replicates.

The adaptive response varied substantially for all populations, ranging from no response at all to almost maximal improvement up to the level of viability in a benign environment. There are several possible explanations for such a lack of adaptation, of which the most likely are either the lack of sufficient genetic variation in a population to adapt, or the selection pressure being too low to affect a population where the tolerance level is already high. In addition, six generations of selection might have been too short to adapt, particularly in case of polygenic resistance traits. Since the intensity of the stress was kept constant during the adaptation process, the resulting selection pressure may have been different for each population because the initial tolerance also varied considerably. Thus, populations with low initial tolerance will have experienced much stronger selection than populations with moderate to high initial tolerance levels (see also the next section). On the other hand, populations with a high level of initial tolerance have already attained near maximal fitness and can hardly show any adaptive response.

The fact that differences in initial tolerance both affect the selection pressures and limit the maximal adaptive response presents a general problem for this kind of experiments. To provide more insight into these effects, Fig. 5 shows the relationship between initial tolerance and adaptive response for all populations. For the salt and ethanol treatments, most populations are clustered in the centre of the "adaptation space", indicating moderate to high initial tolerance and the presence of weak to moderate selection pressures. Although the initial tolerance as such leaves some room for fitness improvement, the two traits nevertheless show little adaptive increase of viability, although it is clear that by far the most (sub)populations are distributed above the zero-line. This is consistent with a polygenic architecture underlying these traits, and in line with the simulation results. As selection pressures on individual genes are expected to be small in case of a polygenic architecture, six generations of adaptation might be too short to obtain distinctive results. In this context one should realise that we set stress levels such that the populations were tolerant enough initially to maintain themselves without any adaptation.

The high temperature treatment shows a completely different pattern. A small number of populations (Fig. 5, open circles) with a very low initial tolerance near to the lower boundary of the adaptation space did not improve viability at all, although the selection pressure must have been high for these populations. Even though we have not

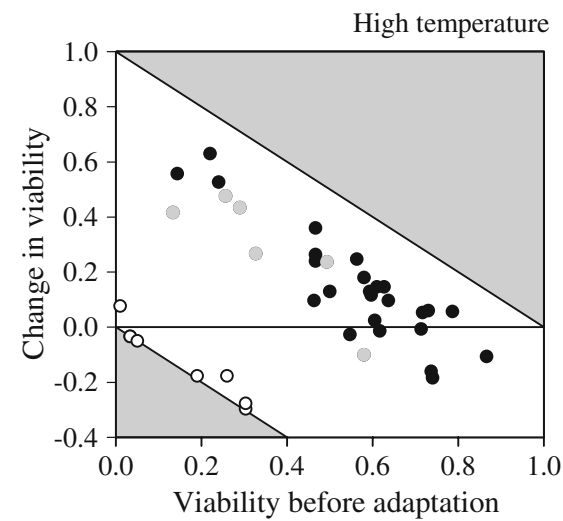

Fig. 5 Relationship between initial tolerance and adaptive response to three stress environments. The white area comprises all possible outcomes of the relationship, and the horizontal lines indicate zero
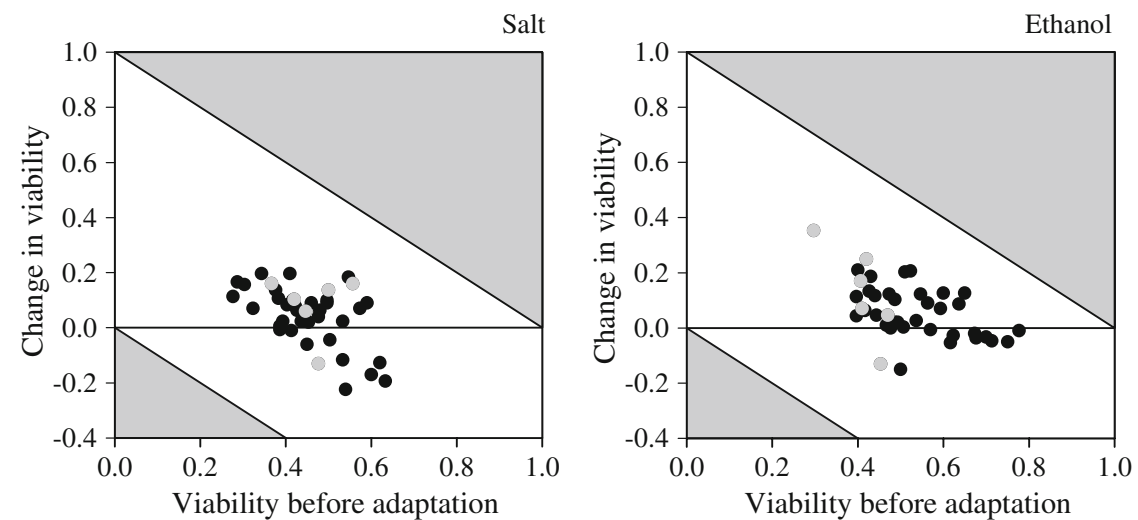

change. Black filled circles indicate demes and grey filled circles indicate undivided populations. Open circles indicate the demes with very low tolerance to high temperature 
done the formal genetics, there is little doubt that these populations had become fixed for the detrimental observed at $29^{\circ} \mathrm{C}$ in the $b w ; s t$ marker stock. One should realize that the conditional lethal initially will have a frequency of around $50 \%$ in the populations and acts as a neutral character at $25^{\circ} \mathrm{C}$. Thus, the probability of fixation for this allele is quite substantial. All other populations are more or less aligned along the upper boundary of the adaptation space, indicating that they had all attained near maximal viability despite considerable variation in initial tolerance. Again, this is most likely explained by assuming that the populations with low initial tolerance had attained high frequencies of the detrimental but had not become fixed for it. In this situation natural selection at high temperature can be very effective and rapidly decrease the frequency of this conditionally expressed detrimental, thereby greatly improving viability. The observed pattern aligns well with the results of the simulations as depicted in the bottom row of Fig. 4.

\section{General implications}

Our study experimentally investigates the effects of population subdivision on the adaptive response for traits with various genetic architectures. One has to realize, however, that the experimental setup we used, i.e., crossing two laboratory stocks to initiate the base population, does not directly mimic the natural situation. For example, the conditional lethal for high temperature may under natural conditions not have reached such a high frequency as in the experiment. Nevertheless, this setup provides good insights into the underlying processes and could be representative, for instance, for the dynamics of disease-sensitive or disease-resistant alleles in situations where the incidence of the disease is rare. As such, our experiment presents relevant information for conservation genetics about the dynamics and consequences of long-term fragmentation for the adaptive potential of species living in a fragmented world.

Our findings with regard to high temperature resistance confirm and extend the conclusion of Reed et al. (2002) that lethal and highly detrimental alleles of large effect, i.e., acting as major genes, are the likely cause of the large lineage effects they observed. We found similar large variation among our experimental demes, but we were also able to distinguish between initial zero tolerance due to fixation of near-lethal deleterious alleles preventing population survival at first exposure to stress, and the adaptive response of the remaining tolerant populations.

We found a clear effect of subdivision on tolerance resulting in large variation among demes instead of within demes due to increased genetic differentiation (i.e., redistribution of genetic variation), which is supported by the results from simulations. In practice, such large variation among demes implies that many demes within a metapopulation are potentially at risk from a lack of adaptive potential, or more importantly, from having become fixed for potentially detrimental or even lethal alleles, whereas none of the undivided populations did become fixed for these alleles in the same time frame. The results for high temperature stress where fixation of conditionally expressed detrimental alleles might lead to near-zero tolerance, and thus, population extinction at first exposure, illustrate the importance of the genetic architecture of a trait for its potential adaptive response. Unlike normally expressed detrimental alleles of large effect that are generally efficiently purged from small populations (Hedrick 1994; Wang et al. 1999; Glemin 2003), conditionally expressed alleles of large effect may have unexpected, negative effects in small populations (Ross-Gillespie et al. 2007). The simulation results indicate that this is of particular importance when migration rates are too low to mitigate the loss of genetic diversity due to allele fixation. The simulation results also indicated that the adaptive response is generally larger on average for undivided than for subdivided populations because there is no genetic differentiation in the former, although we did not always observe this in the experimental situation where undivided populations occasionally showed lower initial tolerance and adaptive response (see below). These predictions are in accordance with the expectations from the literature on bottlenecked populations (Whitlock and Fowler 1999; Saccheri et al. 2001; Swindell and Bouzat 2005; Briggs and Goldman 2006). Our experimental data also confirm this (see Fig. 3, right column, bottom panel), although the differences were found to be not significant (except when the data were combined over stresses). This is generally one of the problems when dealing with bottlenecked populations, as this process will necessarily increase the variance among demes and populations.

From a conservation perspective, this paper highlights two important issues. First, long-term fragmentation in combination with restricted gene flow will limit the adaptive potential of individual subpopulations because adaptive variation will become distributed among (sub)populations rather than within (sub)populations. This is true in general, but as this process is stochastic there may always be exceptions. Consequently, (sub)populations become pauperized of standing genetic variation that is prerequisite for successful adaptation to novel environments (Bell and Gonzalez 2009). Promoting sufficient gene flow between (sub)populations is therefore an important management measure as this may restore the adaptive potential (Swindell and Bouzat 2006). This is the more important because many endangered species are currently subject to changing and deteriorating environmental conditions. 
Second, the genetic architecture of the trait(s) under selection is of great significance to understand the possible responses to novel stresses that one might expect. Traits governed by major genes (e.g., disease resilience genes, resistance to chemical pollutants and conditionally expressed deleterious alleles) will exhibit a large variance in tolerance among (sub)populations and upon first exposure to a novel stress many (sub)populations may readily go extinct while others are only moderately affected by the stress especially when selection pressures are high. However, if the (sub)populations are still variable for the right alleles, they will be able to adapt quickly to the new conditions. In contrast, polygenic traits will show much less variation in tolerance among (sub)populations and most will exhibit a moderate tolerance upon first exposure. However, their adaptive response may be much less and slower. This may in fact endanger the persistence of the (sub)populations to a large extent when the rate of environmental change is high.

Acknowledgements We thank M.H. Edelenbos, A. Boerema and R. Houwerzijl for their technical assistance. We thank Torsten N. Kristensen and two anonymous reviewers for their constructive comments on earlier versions of the manuscript. J.B. was financially supported by the Netherlands Foundation for the Advancement of Tropical Research (WOTRO), as part of the Priority Program "Biodiversity in Disturbed Ecosystems" of the Netherlands Organization for Scientific Research (NWO). M.E.C.v.R. was financially supported by the graduate school Functional Ecology as part of the "Breedtestrategie" programme "Dispersal and Adaptation". This paper profited from the stimulating discussions within the framework of the ESF Scientific Networking Programme ConGen.

Open Access This article is distributed under the terms of the Creative Commons Attribution Noncommercial License which permits any noncommercial use, distribution, and reproduction in any medium, provided the original author(s) and source are credited.

\section{References}

Armbruster P, Reed DH (2005) Inbreeding depression in benign and stressful environments. Heredity 95:235-242

Bell G, Gonzalez A (2009) Evolutionary rescue can prevent extinction following environmental change. Ecol Lett 12:942-948

Bijlsma R, Loeschcke V (2005) Environmental stress, adaptation and evolution: an overview. J Evol Biol 18:744-749

Bijlsma R, Bundgaard J, Van Putten WF (1999) Environmental dependence of inbreeding depression and purging in Drosophila melanogaster. J Evol Biol 12:1125-1137

Bijlsma R, Bundgaard J, Boerema AC (2000) Does inbreeding affect the extinction risk of small populations? Predictions from Drosophila. J Evol Biol 13:502-514

Bijlsma-Meeles E (1979) Viability in Drosophila melanogaster in relation to age and $\mathrm{ADH}$ activity of eggs transferred to ethanol food. Heredity 42:79-89

Briggs WH, Goldman IL (2006) Genetic variation and selection response in model breeding populations of Brassica rapa following a diversity bottleneck. Genetics 172:457-465
Bryant EH, Meffert LM (1996) Nonadditive genetic structuring of morphometric variation in relation to a population bottleneck. Heredity 77:168-176

Bryant EH, McCommas SA, Combs LM (1986) The effect of an experimental bottleneck upon quantitative genetic variation in the housefly. Genetics 114:1191-1211

Cavicchi S, Guerra D, Latorre V, Huey RB (1995) Chromosomal analysis of heat-shock tolerance in Drosophila melanogaster evolving at different temperatures in the laboratory. Evolution 49:676-684

Chakir M, Capy P, Genermont J, Pla E, David JR (1996) Adaptation to fermenting resources in Drosophila melanogaster: ethanol and acetic acid tolerances share a common genetic basis. Evolution 50:767-776

Cheverud JM, Vaughn TT, Pletscher LS, King-Ellison K, Bailiff J, Adams E, Erickson C, Bonislawski A (1999) Epistasis and the evolution of additive genetic variance in populations that pass through a bottleneck. Evolution 53:1009-1018

Crnokrak P, Roff DA (1999) Inbreeding depression in the wild. Heredity 83:260-270

David JR, Allemand R, van Herrewege J, Cohet Y (1983) Ecophysiology: abiotic factors. In: Ashburner H, Carson L, Thompson N (eds) The genetics, biology of Drosophila. Academic Press, London, pp 105-170

Dionne M, Miller KM, Dodson JJ, Bernatchez L (2009) MHC standing genetic variation and pathogen resistance in wild Atlantic salmon. Philos Trans R Soc B Biol Sci 364:1555-1565

Dobzhansky T, Pavlovsky O, Spassky B, Spassky N (1955) Genetics of natural populations 23. Biological role of deleterious recessives in populations of Drosophila pseudoobscura. Genetics 40:781-796

England PR, Osler GHR, Woodworth LM, Montgomery ME, Briscoe DA, Frankham R (2003) Effects of intense versus diffuse population bottlenecks on microsatellite genetic diversity and evolutionary potential. Conserv Genet 4:595-604

Frankham R (2005) Genetics and extinction. Biol Conserv 126: $131-140$

Frankham R, Lees K, Montgomery ME, England PR, Lowe EH, Briscoe DA (1999) Do population size bottlenecks reduce evolutionary potential? Anim Conserv 2:255-260

Glemin S (2003) How are deleterious mutations purged? Drift versus nonrandom mating. Evolution 57:2678-2687

Hedrick PW (1994) Purging inbreeding depression and the probability of extinction: full-sib mating. Heredity 73:363-372

Krebs RA, LaTorre V, Loeschcke V, Cavicchi S (1996) Heat-shock resistance in Drosophila populations: analysis of variation in reciprocal cross progeny. Hereditas 124:47-55

Kristensen TN, Sørensen AC, Sorensen D, Pedersen KS, Sørensen JG, Loeschcke V (2005) A test of quantitative genetic theory using Drosophila - effects of inbreeding and rate of inbreeding on heritabilities and variance components. J Evol Biol 18: $763-770$

Kristensen TN, Barker JSF, Pedersen KS, Loeschcke V (2008) Extreme temperatures increase the deleterious consequences of inbreeding under laboratory and semi-natural conditions. Proc R Soc Lond B Biol Sci 275:2055-2061

Lindsley DL, Grell EH (1968) Genetic variations of Drosophila melanogaster. Carnegie Inst. Wash. Publ. No. 627. Carnegie Institution, Washington, DC

Loeschcke V, Krebs RA, Dahlgaard J, Michalak P (1997) Hightemperature stress and the evolution of thermal resistance in Drosophila. In: Bijlsma R, Loeschcke V (eds) Environmental stress adaptation and evolution. Birkhauser Verlag, Basel, pp 175-190

Lynch M, Lande R (1993) Evolution and extinction in response to environmental change. In: Kareiva PM, Kingsolver JG, Huey RB 
(eds) Biotic interactions, global change. Sinauer Associates, Sunderland, pp 234-250

Macnair MR (1991) Why the evolution of resistance to anthropogenic toxins normally involves major gene changes-the limits to natural selection. Genetica 84:213-219

Malherbe Y, Kamping A, Van Delden W, Van de Zande L (2005) ADH enzyme activity and Adh gene expression in Drosophila melanogaster lines differentially selected for increased alcohol tolerance. J Evol Biol 18:811-819

Orr HA, Unckless RL (2008) Population extinction and the genetics of adaptation. Am Nat 172:160-169

Oudman L, Van Delden W, Kamping A, Bijlsma R (1991) Polymorphism at the $A d h$ and $\alpha G p d h$ loci in Drosophila melanogaster-effects of rearing temperature on developmental rate, body weight, and some biochemical parameters. Heredity 67:103-115

Reed DH, Briscoe DA, Frankham R (2002) Inbreeding and extinction: the effect of environmental stress and lineage. Conserv Genet 3:301-307

Reed DH, Lowe EH, Briscoe DA, Frankham R (2003) Fitness and adaptation in a novel environment: effect of inbreeding, prior environment, and lineage. Evolution 57:1822-1828

Reed DH, Nicholas AC, Stratton GE (2007) Inbreeding levels and prey abundance interact to determine fecundity in natural populations of two species of wolf spider. Conserv Genet 8:1061-1071

Rohmer C, David JR, Moreteau B, Joly D (2004) Heat induced male sterility in Drosophila melanogaster: adaptive genetic variations among geographic populations and role of the $\mathrm{Y}$ chromosome. J Exp Biol 207:2735-2743

Ross-Gillespie A, O'Riain MJ, Keller LF (2007) Viral epizootic reveals inbreeding depression in a habitually inbreeding mammal. Evolution 61:2268-2273

Ruano RG, Silvela LS, LopezFanjul C, Toro MA (1996) Changes in the additive variance of a fitness-related trait with inbreeding in Tribolium castaneum. J Anim Breed Genet Z Tierz Zungt Biol 113:93-97

Saccheri I, Kuussaari M, Kankare M, Vikman P, Fortelius W, Hanski I (1998) Inbreeding and extinction in a butterfly metapopulation. Nature 392:491-494

Saccheri IJ, Nichols RA, Brakefield PM (2001) Effects of bottlenecks on quantitative genetic variation in the butterfly Bicyclus anynana. Genet Res 77:167-181
Sokal RR, Rohlf FJ (1995) Biometry, 3rd edn. W.H. Freeman, New York

Sorensen JG, Nielsen MM, Kruhoffer M, Justesen J, Loeschcke V (2005) Full genome gene expression analysis of the heat stress response, in Drosophila melanogaster. Cell Stress Chaperones 10:312-328

Suzuki DT (1970) Temperature-sensitive mutations in Drosophila melanogaster. Science 170:695-706

Swindell WR, Bouzat JL (2005) Modeling the adaptive potential of isolated populations: experimental simulations using Drosophila. Evolution 59:2159-2169

Swindell WR, Bouzat JL (2006) Gene flow and adaptive potential in Drosophila melanogaster. Conserv Genet 7:79-89

Tobari I (1966) Effects of temperature on viability of heterozygotes of lethal chromosomes in Drosophila melanogaster. Genetics 53:249-259

Van Delden W, Kamping A (1979) Alcohol dehydrogenase polymorphism in populations of Drosophila melanogaster. 3. Differences in developmental times. Genet Res 33:15-27

Vermeulen CJ, Bijlsma R (2004) Characterization of conditionally expressed mutants affecting age-specific Drosophila melanogaster: lethal conditions and temperature-sensitive periods. Genetics 167:1241-1248

Vermeulen CJ, Bijlsma R, Loeschcke V (2008) A major QTL affects temperature sensitive adult lethality and inbreeding depression in life span in Drosophila melanogaster. BMC Evol Biol 8:297

Wallace B (1982) Drosophila melanogaster populations selected for resistance to $\mathrm{NaCl}$ and $\mathrm{CuSO}_{4}$ in both allopatry and sympatry. J Hered 73:35-42

Wang JL, Hill WG, Charlesworth D, Charlesworth B (1999) Dynamics of inbreeding depression due to deleterious mutations in small populations: mutation parameters and inbreeding rate. Genet Res 74:165-178

Whitlock MC (2002) Selection, load and inbreeding depression in a large metapopulation. Genetics 160:1191-1202

Whitlock MC, Fowler K (1999) The changes in genetic and environmental variance with inbreeding in Drosophila melanogaster. Genetics 152:345-353

Willi Y, Hoffmann AA (2009) Demographic factors and genetic variation influence population persistence under environmental change. J Evol Biol 22:124-133

Wright S (1951) The genetical structure of populations. Ann Eugen 15:323-354 